\title{
ВОЗРАСТ-ОБУСЛОВЛЕННОЕ ИЗМЕНЕНИЕ МИКРОЭЛЕМЕНТНОГО ПРОФИЛЯ ЛИМФОУЗЛОВ С УЧЕТОМ ИХ ЛОКАЛИЗАЦИИ
}

\author{
В.Н. Горчаков ${ }^{1 *}$, Ю.П. Колмогоров ${ }^{2}$ О.В. Горчакова ${ }^{2}$ \\ ${ }^{1}$ Новосибирский государственный университет, 630090, Новосибирск, ул. Пирогова, 2 \\ ${ }^{2}$ Научно-исследовательский институт клинической и экспериментальной лимфологии - филиал ИЦиГ СО РАН, \\ 630060, Новосибирск, ул. Тимакова, 2 \\ *e-mail: vgorchak@yandex.ru
}

РЕЗЮМЕ. Исследовано содержание микроэлементов в регионарных лимфоузлах с учетом локализации и возраста при помощи рентгенфлюоресцентного анализа с использованием синхротронного излучения. Микроэлементный профиль лимфоузлов носит регион-зависимый характер, отражая своеобразие лимфатического региона. Различия в содержании микроэлементов определяют микроэлементный профиль каждого из регионарных лимфоузлов. Брыжеечный лимфоузел имеет максимальное содержание $\mathrm{Cu}, \mathrm{Se}$, среднее - Fe, Zn, минимальное $\mathrm{Mn}$. Трахеобронхиальный лимфоузел характеризуется наименьшим содержанием $\mathrm{Fe}, \mathrm{Cu}, \mathrm{Zn}, \mathrm{cpeдним} \mathrm{-} \mathrm{Mn,} \mathrm{Se.}$ Паховый лимфоузел отличает максимальное содержание $\mathrm{Mn}, \mathrm{Fe}, \mathrm{Zn}$, среднее - $\mathrm{Cu}$, минимальное - Se. При старении меняется микроэлементый профиль лимфоузлов, демонстрируя уменьшение содержания большинства микроэлементов при увеличении Мn. Формируется качественно новый микроэлементный профиль каждого из лимфоузлов при старении, отражая снижение дренажной и иммунной функции лимфоузлов. С возрастом в паховом лимфоузле имеют место относительно высокие показатели содержания $\mathrm{Mn}, \mathrm{Fe}, \mathrm{Zn}$ и наименьшее - для Сu и Se. Брыжеечный лимфоузел отличает высокое значение концентрации $\mathrm{Se}$, среднее - $\mathrm{Cu}, \mathrm{Zn}$ и низкое $\mathrm{Mn}, \mathrm{Fe}$. Трахеобронхиальный лимфоузел характеризуется низким значением содержания Zn, средним - Mn, Fe, Se и высоким - Cu. Полученные результаты доказывают регионарную специфику микроэлементного содержания лимфоузлов в зависимости от региона и возраста.

КЛЮЧЕВЫЕ СЛОВА: микроэлементы, лимфоузлы, онтогенез, геронтология.

\section{AGE-CAUSED CHANGE OF THE MICROELEMENT PROFILE OF LYMPH NODES OF DIFFERENT LOCALIZATION}

\author{
V.N. Gorchakov ${ }^{1 *}$, Yu.P. Kolmogorov ${ }^{2}$, O.V. Gorchakova ${ }^{2}$ \\ ${ }^{1}$ Novosibirsk State University, 630090, Novosibirsk, Pirogov str., 2 \\ ${ }^{2}$ Research Institute of clinical and experimental Lymphology - branch of Institute of Cytology and Genetics \\ of the Siberian Branch of The Russian Academy of Science, 630060, Novosibirsk, Timakov str., 2 \\ *e-mail: vgorchak@yandex.ru
}

\begin{abstract}
The purpose is a research of content of microelements in regional lymph nodes in view of localization and age. Microelements defined with the help X-ray fluorescent analysis with use of synchrotron radiation. The microelement profile of lymph nodes has the region-dependent character, reflecting an originality of the lymphatic region. Distinctions of microelements content are recorded for each of regional lymph nodes. The mesenteric lymph node has the maximum maintenance of $\mathrm{Cu}$, Se, an average - Fe, $\mathrm{Zn}$, minimum - Mn. The tracheobronchial lymph node is characterized by the smallest maintenance of $\mathrm{Fe}, \mathrm{Cu}, \mathrm{Zn}$, an average - Mn, Se. The inguinal lymph node distinguishes the maximum content of $\mathrm{Mn}, \mathrm{Fe}, \mathrm{Zn}$, an average - $\mathrm{Cu}$, minimum - Se. When aging the microelement profile of lymph nodes changes, showing reduction of content of the majority of microelements at increase in Mn. Qualitatively new microelement profile is formed in each of lymph nodes when aging. It reflects decrease in drainage and immune function of lymph nodes at the scarce level of bioelements. With age in the inguinal lymph node rather high rates of content of $\mathrm{Mn}, \mathrm{Fe}, \mathrm{Zn}$ and the smallest take place for $\mathrm{Cu}$ and Se. The mesenteric lymph node distinguishes high value of concentration $\mathrm{Se}$, an average $-\mathrm{Cu}, \mathrm{Zn}$ and low $-\mathrm{Mn}, \mathrm{Fe}$. The tracheobronchial lymph node is characterized by low value of contents $\mathrm{Zn}$, an average - Mn, Fe, Se and high $-\mathrm{Cu}$. The received results prove regional specifics of microelement balance of lymph nodes depending on the region and age.
\end{abstract}

KEYWORDS: trace elements, lymph nodes, ontogenesis, gerontology.

\section{ВВЕДЕНИЕ}

Одним из важных регуляторов гомеостаза многих систем организма является полноценный состав микроэлементов, участвующих в механизмах иммунного ответа и функционирования лимфоидной 
системы (Скальный и др., 2004; Бородин и др., 2018; Василенко и др., 2019). С возрастом меняются не только лимфоузлы, но и биоэлементный обмен, что лимитирует характер адаптивных и патологических перестроек в органах. Лимфоузлы выполняют иммунную и дренажную функции, обеспечивая регионарный гомеостаз и защиту в разные периоды жизни (Кудрин и др., 2000; Бородин и др., 2018). Выполнение функции лимфоузлов не может обойтись без микроэлементов, которые аккумулируются в лимфоузлах. Микроэлементы, изменяя активность ферментов, влияют на пролиферацию и дифференцировку клеток лимфоидной ткани, что формирует оптимальный иммунный ответ в лимфатическом регионе (Nomura et al., 2002; Intorre et al., 2008; Василенко и др., 2019). Структурно и функционально лимфоузлы привязаны к территории, которую дренируют, что находится в соответствии с положениями концепции лимфатического региона (Suami, 2017; Бородин и др., 2018). Это делает актуальным необходимость изучения микроэлементного статуса лимфоузлов в зависимости от $\begin{array}{lllll}\text { локализации и } & \text { и } & \text { вчетом } & \text { возрастного }\end{array}$ В литературе недостаточно информации о возрастной динамике микроэлементного статуса лимфоузлов, принадлежащих к разным лимфатическим регионам. Такое исследование имеет теоретический и практический интерес.

Цель исследования - изучить содержание микроэлементов в регионарных лимфоузлах с учетом их локализации и возраста в эксперименте.

\section{МАТЕРИАЛЫ И МЕТОДЫ}

В эксперименте участвовали 120 белых крыс Wistar в возрасте 3-5 месяцев (период максимального развития лимфоидной ткани) и 1,5-2 года (период инволюция лимфоидной ткани). Условно выделены группы молодых и старых животных соответственно. Объектом исследования были брыжеечные, паховые и трахеобронхиальные лимфоузлы, принадлежащие к разным лимфатическим регионам. Содержание микроэлементов ( $\mathrm{Se}, \mathrm{Mn}, \mathrm{Fe}, \mathrm{Cu}, \mathrm{Zn}$ ) в лимфоузлах определяли с помощью рентгенфлуоресцентного анализа с использованием синхротронного излучения (РФА СИ). В работе использовали оборудование ЦКП «СЦСТИ» на базе УНУ «Новосибирский ЛСЭ» / «Комплекс ВЭПП-4 ВЭПП-2000» в Институте ядерной физики СО РАН (Piminov, 2016). Обработку данных выполняли с помощью лицензированной статистической программы StatPlus Pro, AnalystSoff Inc.

\section{РЕЗУЛЬТАТЫ И ОБСУЖДЕНИЕ}

Для поддержания структурной организации лимфоузлов в молодом возрасте необходимы микроэлементы для дифференцировки и пролиферации иммунокомпетентных клеток, участвующих в формировании функционально-значимых компартментов лимфоузлов (Gorchakova et al., 2019). Микроэлементный профиль лимфоузлов носит регион-зависимый характер, отражая своеобразие лимфатического региона.

Для молодых животных считается оптимальным содержание микроэлементов в лимфоузлах для обеспечения их функции, когда лимфоидная ткань достигает максимального развития (Бородин и др., 2018). У молодых животных в паховом лимфоузле содержание $\mathrm{Mn}$ составляет 4,12 $\pm 0,32$ мкг/г $(p<0,001)$, что превышает в $1,6-1,9$ раза показатели в трахеобронхиальном $(2,54 \pm 0,15$ мкг/г) и брыжеечном $(2,15 \pm 0,13$ мкг/г) лимфоузлах. Содержание $\mathrm{Fe}$ в паховом лимфоузле составляет $672,55 \pm 54,22$ мкг/г $(p<0,001)$, что превышает в $2,6-3,0$ раза показатели в брыжеечном $(254,82 \pm 20,66$ мкг/г) и трахеобронхиальном $(221,38 \pm 12,12$ мкг/г) лимфоузлах, Содержание $\mathrm{Cu}$ в паховом лимфоузле составляет $6,45 \pm 0,35$ мкг/г, что превышает в 1,2 раза показатель в трахеобронхиальном $(5,27 \pm 0,17$ мкг/г; $p<0,01)$ и не дает статистического различия с показателем в брыжеечном $(6,48 \pm 0,47$ мкг/г; $p>0,05)$

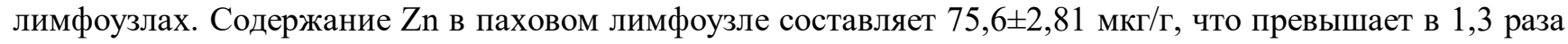
показатель в трахеобронхиальном $(58,26 \pm 2,30$ мкг $/ 5 ; p<0,001)$ и не дает статистического различия с показателем в брыжеечном $(68,71 \pm 2,52$ мкг/г; $p>0,05)$ лимфоузлах. При этом существует статистически значимая разница по содержанию $\mathrm{Zn}$ между трахеобронхиальном и брыжеечным лимфоузлами ( $p<$ $0,01)$. Содержание Se наименьшее в паховом лимфоузле и составляет $0,96 \pm 0,05$ мкг/г, что меньше в $1,3-$ 
1,4 раза его содержания в трахеобронхиальном $(1,25 \pm 0,06$ мкг/г; $p<0,01)$ и брыжеечном $(1,38 \pm 0,05$ мкг/г; $p<0,001)$ лимфоузлах соответственно.

Выявленные различия в содержании микроэлементов определяют микроэлементный профиль каждого из регионарных лимфоузлов. Так, в брыжеечном лимфоузле имеет место максимальное содержание $\mathrm{Cu}, \mathrm{Se}$, среднее - Fe, $\mathrm{Zn}$, минимальное - Mn. Трахеобронхиальный лимфоузел характеризуется наименьшим содержанием $\mathrm{Fe}, \mathrm{Cu}, \mathrm{Zn}$, средним - Mn, Se. Паховый лимфоузел отличает максимальное содержание $\mathrm{Mn}, \mathrm{Fe}, \mathrm{Zn}$, среднее - $\mathrm{Cu}$, минимальное $-\mathrm{Se}$. Очевидно, что различие в содержании микроэлементов связано с особенностями дренируемого лимфатического региона и разным вкладом биоэлементов в его функционирование на уровне лимфоузлов.

Общей закономерностью старения является инволюция лимфоидной ткани с нарушением гомеостаза биоэлементов (Кудрин и др., 2000; Скальный, Рудаков, 2004; Gorchakova et al., 2019]. В лимфоузлах разных топографических групп отмечено уменьшение содержания большинства микроэлементов $(\mathrm{Zn}, \mathrm{Se}, \mathrm{Cu}, \mathrm{Fe})$ в $1,2-1,5$ раза $(p<0,05-0,01)$ при увеличении содержания $\mathrm{Mn}($ в 1,3 раза, $p<0,05)$ у старых животных в сравнении с молодыми. Это формирует качественно новый микроэлементный профиль при дефицитном содержании большинства микроэлементов в старческом возрасте. Закономерности распределения содержания микроэлементов в различных лимфоузлах старых животных показали, что в паховом лимфоузле наиболее высокие показатели содержания $\mathrm{Mn}, \mathrm{Fe}, \mathrm{Zn}$ и наименьшее - $\mathrm{Cu}$ и $\mathrm{Se}$; в брыжеечном лимфоузле имеет место высокое значение концентрация $\mathrm{Se}$, среднее - $\mathrm{Cu}, \mathrm{Zn}$ и низкое $\mathrm{Mn}, \mathrm{Fe}$; в трахеобронхиальном лимфоузле - низкое значение содержания $\mathrm{Zn}$, среднее - Mn, Fe, Se и высокое значение - $\mathrm{Cu}$. Уровень содержание меди находится в интервале $4,68 \pm 0,27-5,37 \pm 0,14$ мкг/г $(p>0,05)$ в лимфоузлах разных топографических групп. Предполагаем, что сформированный микроэлементный профиль отражает снижение пролиферации лимфоидных клеток и, следовательно, функции лимфоузлов при старении. При этом Mn рассматривается как фактор защиты от перекисных окислений и дегидратации и повышает иммунную функцию (Кудрин и др., 2000; Maggini et al., 2007), что важно для лимфоузлов, подвергнутых процессу старения.

\section{ВЫВОДЫ}

Оптимальное содержание микроэлементов у молодых животных перераспределяется между лимфоузлами с формированием индивидуального микрожлементного профиля, что отражает структурно-функциональные особенности соматического, бронхолегочного, кишечного лимфатических регионов. При старении формируется качественно новый профиль микроэлементов из-за развития их общего дефицита. Содержание микроэлементов в региональных лимфоузлах старых животных перераспределяется иначе, чем у молодых животных. Это компенсирует снижение функции лимфоузлов, подвергнутых старению. Полученные результаты подтверждают реализацию принципа региональной детерминанты в отношении микроэлементного баланса лимфоузлов в разные периоды жизни.

\section{Список литературы}

1. Бородин Ю.И., Горчакова О.В., Суховершин А.В., Горчаков В.Н. и др. Концепция лимфатического региона в профилактической лимфологии. LAP LAMBERT Academic Publishing. 2018. 74 c.

2. Василенко А.М., Шарипова М.М. Дефицит микроэлементов и проблема коморбидности. Микроэлементы в медицине. 2019; 20(1): 4-12. DOI: 10.19112/2413-6174-2019-20-1-4-12].

3. Кудрин А.В., Скальный А.В., Жаворонков А.А., Скальная М.Г. Иммунофармакология микроэлементов. Москва: КМК. 2000. $537 \mathrm{c}$.

4. Скальный А.В., Рудаков Н.А. Биоэлементы в медицине. М.: Издательство: Оникс 21 век, Мир. 2004. 272 с.

5. Gorchakova O., Kolosova N., Gorchakov V., Starkova E., Demchenko G. Premature aging and structural organization of the mesenteric lymph node. Archiv Euromedica. 2019; 9(3): 22-24. https://doi.Org/10.35630/2199-885X/2019/9/3.7

6. Intorre F., Polito A., Andriollo-Sanchez M. Effect of zinc supplementation on vitamin status of middle-aged and older European adults: the ZENITH study. Eur. J. Clin. Nutr. 2008; 62: 1215-1223.

7. Maggini S., Wintergerst E.S., Beveridge S., Hornig D.H. Selected vitamins and trace elements support immune function by strengthening epithelial barriers and cellular and humoral immune responses. Br. J. Nutr. 2007; 98(Suppl 1): $29-35$. DOI: $10.1017 /$ S0007114507832971. 
8. Nomura A., Sugiura $Y$. Contribution of individual zinc ligands to metal binding and peptide folding of zinc finger peptides. Inorg. Chem. 2002; 41: 3693-3698.

9. Piminov P.A. Synchrotron Radiation Research and Application at VEPP-4. Physics Procedia. 2016; 84: 19-26. DOI:10.1016/j.phpro.2016.11.005.

10. Suami H. Lymphosome concept: Anatomical study of the lymphatic system. Journal of Surgical Oncology. 2017; 115(1): 1-5. DOI: $10.1002 /$ jso. 24332 .

\section{References}

1. Borodin Yu.I., Gorchakova O.V., Sukhovershin A.V., Gorchakov V.N., etc. [The concept of the lymphatic region in a preventive lymphology]. LAP LAMBERT Academic Publishing. 2018. 74p.

2. Vasilenko A.M., Sharipova M.M. [Micronutrient deficiency and problem of a komorbidnost]. Microelements in medicine. 2019; 20(1). P. 4-12. DOI: 10.19112/2413-6174-2019-20-1-4-12].

3. Kudrin A.V., Skalnyi A.V., Zhavoronkov A.A., Skalnaja M.G. [Immunopharmacology of microelements]. Moscow: KMK, 2000.537 p.

4. Skalnyi A.V., Rudakov N.A. [Bioelements in medicine]. Moscow: Publishing house "Onyx the 21st century", "World". 2004.272 p.

5. Gorchakova O., Kolosova N., Gorchakov V., Starkova E., Demchenko G. Premature aging and structural organization of the mesenteric lymph node. Archiv Euromedica. 2019; 9(3): 22-24. https://doi.Org/10.35630/2199-885X/2019/9/3.7.

6. Intorre F., Polito A., Andriollo-Sanchez M. Effect of zinc supplementation on vitamin status of middle-aged and older European adults: the ZENITH study. Eur. J. Clin. Nutr. 2008: 62: 1215-1223.

7. Maggini S., Wintergerst E.S., Beveridge S., Hornig D.H. Selected vitamins and trace elements support immune function by strengthening epithelial barriers and cellular and humoral immune responses. Br J Nutr. 2007; 98(Suppl 1): 29-35. DOI: $10.1017 /$ S0007114507832971.

8. Nomura A., Sugiura Y. Contribution of individual zinc ligands to metal binding and peptide folding of zinc finger peptides. Inorg. Chem. 2002; 4: 3693-3698.

9. Piminov P.A. Synchrotron Radiation Research and Application at VEPP-4. Physics Procedia. 2016; 84: 19-26. DOI: 10.1016/j.phpro.2016.11.005.

10. Suami H. Lymphosome concept: Anatomical study of the lymphatic system. Journal of Surgical Oncology. 2017; 115(1): 1-5. DOI: $10.1002 /$ jso. 24332 . 